1. Richard S. Varga, Matrix Iterative Analysis, Prentice-Hall, Inc., 1962.

2. E. G. D'yakonov, "On a Method of Solving the Poisson Equation," Dokl. Akad. Nauk SSSR 143 (1962), 21-24, the same paper appears in English in Soviet Math.-Doklady 3, 1962, p. $320-323$.

3. R. B. Kellogg, "Another Alternating-Direction-Implicit Method," to appear in $J$. Soc. Ind. Appl. Math.

4. A. M. Ostrowski, "Iterative Solution of Linear Systems of Functional Equations," J. Math. Anal. Appl., 2, 1961, p. 351-369.

5. L. Collatz, "Fehlerabschätzung für das Iterationsverfahren zur Auflösung linearer Gleichungssysteme," Z. Angew. Math. Mech., 22, 1942, p. 357-361.

6. DAvid G. Feingold, \& R. S. VARGa, "Block diagonally dominant matrices and generalizations of the Gerschgorin circle theorem," to appear in the Pacific J. Math.

7. J. Douglas, JR., and H. H. RAChFord, JR., "On the numerical solution of heat conduction problems in two or three space variables," Trans. Amer. Math. Soc., 82, 1956, p. 421-439.

8. J. Douglas, JR., "Alternating direction methods for three space variables," Numer. Math., 4,1962 , p. 41-63.

\title{
Generation of Permutations by Adjacent Transposition
}

\author{
By Selmer M. Johnson
}

1. Introduction. In D. H. Lehmer's review [1] of a paper by Mark Wells [2], "Generation of Permutations by Transposition," he states:

"The author describes a new systematic method of generation permutations with the interesting feature that each permutation is derived from its predecessor by a single interchange of two marks. In more than half the cases the two marks are adjacent."

In the present paper a different method is described in which each permutation is derived from its predecessor by a single interchange of two marks in adjacent positions. Moreover, the rules are extremely simple.

First the method will be described in terms of the marks themselves. Then the method will be restated in terms of positions.

2. The Method in Terms of Marks. Define indices $I(k)$ for $k=1,2, \cdots, n$, where $I(k)$ is 0 or 1 according as the permutation on the marks $1,2,3, \cdots, k-1$ is even or odd. By convention, let $I(1)=I(2)=0$. Define $T(k)$ to be the interchange of the mark $k$ with some smaller mark immediately to the left [right] according as $I(k)$ is $0[1]$.

Our rules for generating the next permutation are as follows:

1) At each stage apply $T(m)$, where $m$ is the largest mark for which $T(m)$ is defined.

2) Change the indices $I(k)$ for $m<k \leqq n$.

Repeat the cycle of steps on the new permutation, etc.

Note that according to these rules we apply $T(m)$ only when all larger marks are at the extreme left or right of the set of marks $(1,2, \cdots, m)$ in some order.

It is clear that this method will generate all $n$ ! permutations on $n$ marks once and only once. For each fixed permutation on the marks from 1 to $k$ we move the mark $k+1$ in one direction through every possible position, thus giving each

Received July 6, 1962. 
permutation on $k+1$ marks once and only once provided we have every permutation on $k$ marks once and only once. Since the method can be verified for $k=2$ or 3 , the induction is complete.

It will be noted that the process will automatically stop at the permutation 2,1 , $3,4,5,6, \cdots, n$ since there is no legal move left.

3. The Method in Terms of Positions. The above method can be restated for a computing machine by determining the left-hand position of the adjacent pair of positions whose marks are to be interchanged.

Let $n>1$. Let $P_{N}$ be the $N$ th permutation starting with $P_{0}=1,2,3, \cdots, n$.

Let $N>0$ and

$$
N=n !\left[\frac{d_{2}}{2 !}+\frac{d_{3}}{3 !}+\cdots \frac{d_{n-1}}{(n-1) !}+\frac{d_{n}}{n !}\right],
$$

where $d_{k}=0,1,2, \cdots$, or $k-1$. By convention, $d_{0}=d_{1}=0$. We indicate this by

$$
N=\left(d_{2}, d_{3}, d_{4}, \cdots, d_{n-1}, d_{n}\right) \text {. }
$$

Starting with

$$
\begin{aligned}
1 & =(0,0,0, \cdots, 0,1) \\
n-1 & =(0,0,0, \cdots, 0, n-1) \\
n & =(0,0,0, \cdots, 1,0) \\
\vdots & \vdots \\
n(n-1)-1 & =(0,0,0, \cdots, n-2, n-1) \\
n(n-1) & =(0,0,0, \cdots, 1,0,0) \\
\vdots & \quad \vdots
\end{aligned}
$$

we end with

$$
n !-1=(1,2,3, \cdots, n-2, n-1) .
$$

Fix $N=\left(d_{2}, d_{3}, d_{4}, \cdots, d_{n-1}, d_{n}\right)$ and let $k$ be the largest subscript for which $d_{k}>0$.

Then to generate $P_{N}$ from $P_{N-1}$ we interchange marks in position $S(N)$ and $S(N)+1$, where

(1) $S(N)=a_{N}(k)+b_{N}(k)$.

Here we have

(2) $a_{N}(k)=k-d_{k}\left[d_{k}\right]$ if $d_{k-1}+(k-1) d_{k-2}$ is even [odd];

(3) $b_{N}(n)=0$;

(4) $b_{N}(n-1)=0[1]$ if $d_{n-1}+(n-1) d_{n-2}$ is even [odd];

for even $k<n-1$,

(5) $b_{N}(k)=0[2]$ if $d_{k}$ is even [odd], and for odd $k<n-1$,

(6) $b_{N}(k)=0[1]$ if $d_{k}+d_{k-1}$ is even [odd].

First we relate the indices on each $k$ with the $d$ 's for each $N$. Letting $I_{N}(k)=$ the index of $k$ for $P_{N}$, we note that, for $k-1$ even,

(7) $I_{N}(k)=0[1]$ if $d_{k-1}$ is even [odd]. 
At the start, both $d_{k-1}$ and $I_{0}(k)=0$ and there is a change in parity on both $d_{k-1}$ and $I_{N}(k)$ at every multiple of $k(k+1) \cdots n$. We see that $d_{k-1}$ runs through an even number of values $0,1,2, \cdots, k-2$, and then repeats the cycle, thus alternating even and odd. Likewise, there is a change in parity on the permutations of the marks $1,2, \cdots, k-1$ for such $N$, so that $I_{N}(k) \neq I_{N-1}(k)$.

For $k-1$ odd, note that $I_{N}(k)$ and $d_{k-1}+d_{k-2}$ change parity every multiple of $k(k+1) \cdots n$ steps since $d_{k-1}$ runs through an odd number of values $0,1,2, \cdots$, $k-2$ and then repeats the cycle. By adding $d_{k-2}$ to $d_{k-1}$, we have a quantity expressed in terms of the $d$ 's for each $N$ which has the same parity as that of $I_{N}(k)$. Here

(8) $I_{N}(k)=0[1]$ if $d_{k-1}+d_{k-2}$ is even [odd].

These two cases can be combined as follows:

(9) $I_{N}(k)=0[1]$ if $d_{k-1}+(k-1) d_{k-2}$ is even [odd].

Next, from the description of the method in the previous section and from the definition of $N$ in terms of the $d$ 's, we observe that for each $N, k$ is the mark to be moved, $a_{N}(k)$ is the left-hand position of the interchange pair relative to the set of marks from 1 to $k, b_{N}(k)$ is the number of marks larger than $k$ currently anchored at the extreme left of the permutation. Thus $b_{N}(k)$ is added to $a_{N}(k)$ to get the correct left-hand position of the interchange pair relative to the complete set of $n$ marks.

To see this, observe that $a_{N}(k)=k-d_{k}$ if the mark $k$ is to be moved to the left, i.e., $I_{N-1}(k)=0=I_{N}(k)$. Also, $a_{N}(k)=d_{k}$ if the mark $k$ is to be moved to the right, i.e., $I_{N-1}(k)=1=I_{N}(k)$. Combining these remarks with (9) gives (2).

Now, turn to $b_{N}(k)$. It is clear that $b_{N}(n)=0$. Also, for $N$ any odd [even] multiple of $n$ we have the mark $n$ at the left [right] with $I_{N-1}(n)=0[1]$. Thus we have $P_{N-1}$ of type either

$$
n^{0},\left(P_{\text {even }}(1,2, \cdots, n-1)\right) \text { or }\left(P_{\text {odd }}(1,2, \cdots, n-1)\right), n^{1},
$$

with even or odd permutations on the marks $1,2, \cdots, n-1$. Here indices are indicated as superscripts.

Then $b_{N}(n-1)=1[0]$ if $I_{N-1}(n)=0[1]$ or if $I_{N}(n)=1[0]$, since there will be a change in the index of $n$ at this stage. These remarks together with (9) give (4).

Next for $k<n-1$ and $N$ an even multiple of $(k+1)(k+2) \cdots n$, note that the mark $k+1$ has made an even number of passages through the smaller marks and is currently at the right of them, as are all the marks $>k+1$. Then $P_{N-1}$ is of the type

$$
\left(P_{\text {odd }}(1,2, \cdots, k)\right), \quad(k+1)^{1}, \quad(k+2)^{1}, \cdots, n^{1},
$$

and since $k$ is moved here to get $P_{N}$ we note that $I_{N-1}(k+1)=1$, so $I_{N}(k+1)=0$. Thus by (7) we have $b_{N}(k)=0$ if $d_{k}$ is even for $k$ even, or if $d_{k}+d_{k-1}$ is even for $l$ odd by (8).

For $N$ an odd multiple of $(k+1)(k+2) \cdots n$ and odd $k<n-1$, the mark $k+1$ has made an odd number of passages through the smaller marks and is then at the left with $I_{N-1}(k+1)=0$. Since $k$ is odd, however, the permutation on the marks $1,2, \cdots, k+1$ is odd, so that $k+2$ is at the right of these marks with $I_{N-1}(k+2)=1$, as are all other marks $>k+2$. Thus we have a permutation 
$P_{N-1}$ of the type

$$
(k+1)^{0}, \quad\left(P_{\text {even }}(1,2, \cdots, k)\right), \quad(k+2)^{1}, \quad(k+3)^{1}, \cdots,(n)^{1} .
$$

Thus $b_{N}(k)=1$ if $k$ is odd and $I_{N-1}(k+1)=1$, i.e., $I_{N}(k+1)=0$. By $(8)$, we have $(6)$.

Finally, for even $k<n-1$ and $N$ an odd multiple of $(k+1)(k+2) \cdots n$, the mark $k+1$ will have made an odd number of passages through the smaller marks and will be at the left of an even permutation of the marks from 1 to $k$. Thus the permutation of the marks from 1 to $k+1$ is also even since $k$ is even and so $I_{N-1}(k+2)=0$ with $k+2$ at the extreme left. The permutation on the marks $1,2, \cdots, k+2$ is then odd, so that $k+3$ is at the right of these marks with $I_{N-1}(k+3)=1$, as are the marks larger than $k+3$. The permutation $P_{N-1}$ is of the type

$$
(k+2)^{0}, \quad(k+1)^{0}, \quad\left(P_{\text {even }}(1,2, \cdots, k)\right), \quad(k+3)^{1}, \quad(k+4)^{1}, \cdots, n^{1} .
$$

Since $I_{N-1}(k+1)=0, I_{N}(k+1)=1$, or by $(7)$ we see that $b_{N}(k)=2$ for even $k<n-1$ and $d_{k}$ odd as (5) states.

The following table illustrates the technique as applied to the generation of 24 permutations on 4 marks, reading downward rather than left to right:

$$
\begin{array}{llllllllllllllllllllllll}
1 & 1 & 1 & 4 & 4 & 1 & 1 & 1 & 3 & 3 & 3 & 4 & 4 & 3 & 3 & 3 & 2 & 2 & 2 & 4 & 4 & 2 & 2 & 2 \\
2 & 2 & 4 & 1 & 1 & 4 & 3 & 3 & 1 & 1 & 4 & 3 & 3 & 4 & 2 & 2 & 3 & 3 & 4 & 2 & 2 & 4 & 1 & 1 \\
3 & 4 & 2 & 2 & 3 & 3 & 4 & 2 & 2 & 4 & 1 & 1 & 2 & 2 & 4 & 1 & 1 & 4 & 3 & 3 & 1 & 1 & 4 & 3 \\
4 & 3 & 3 & 3 & 2 & 2 & 2 & 4 & 4 & 2 & 2 & 2 & 1 & 1 & 1 & 4 & 4 & 1 & 1 & 1 & 3 & 3 & 3 & 4
\end{array}
$$

The RAND Corporation

Santa Monica, California

1. D. H. LEHMER, “Generation of permutations by transposition," Math. Reviews, v. 23, 1962, no. 1B, B553, p. 94 .

2. MARK B. Wells, "Generation of permutations by transposition," Math. Comp., v. 15, 1961, p. 192-195.

\title{
Determinants by Means of Solutions of Linear Equations
}

\author{
By R. W. Preisendorfer, B. W. Roos \& W. C. Sangren
}

1. Introduction. In this paper, we are considering the converse of the classical problem of "Solutions of Linear Equations by Means of Determinants." The present problem arises from the occasional need for the explicit value of a determinant associated with a system of equations that is being solved by a machine method which does not necessarily involve the classical Cramer method of solution using determinants. Suppose then that a particular program of computation for the solution of a system of linear equations does not explicitly evaluate the determinant of the matrix of the system, but that nevertheless the determinant for some reason is

Received August 17, 1961, revised October 29, 1962. 